\title{
Suggestion For a New Classification of Postoperative Neurological Complications (CPNC) in Neurosurgery
}

David Shalamberidze ( $\sim$ davidshalamberi@gmail.com )

Klinikum Arnsberg GmbH: Klinikum Hochsauerland GmbH https://orcid.org/0000-0001-8785-5877

Yasar Al Awad

Klinikum Arnsberg GmbH: Klinikum Hochsauerland GmbH

Jürgen Höpfner

Klinikum Arnsberg GmbH: Klinikum Hochsauerland GmbH

Guido Kluge

Klinikum Arnsberg GmbH: Klinikum Hochsauerland GmbH

\section{Ludwig Benes}

Klinikum Arnsberg GmbH: Klinikum Hochsauerland GmbH

\section{Research Article}

Keywords: Classification, Complication, Neurosurgery, Outcome, Neurological Disturbance

Posted Date: March 26th, 2021

DOI: https://doi.org/10.21203/rs.3.rs-353350/v1

License: (c) (i) This work is licensed under a Creative Commons Attribution 4.0 International License. Read Full License 


\section{Abstract}

To design a classification concentrating on the art and severity of functional restriction that could include all possible postoperative neurological disturbances and prove its validity to be comparable and easily used in neurosurgery. A seven grade classification was proposed commencing from grade " 0 " for no neurological disturbances to grade "6" for coma/vegetative state/demise: grade "1", any remittent neurological disturbances with full improvement spontaneously or as a result of any temporary drug therapy or re-surgery, a single epileptic seizure without the need for anticonvulsant therapy; grade " 2 ", lasting neurological disturbances resulting in no functional restrictions, no epileptic seizures needing for long-term anticonvulsant therapy; grade " 3 ", lasting neurological disturbances resulting in slight functional restrictions, rare epileptic seizures despite of anticonvulsant therapy, slight aphasia; grade " 4 ", lasting neurological disturbances resulting in moderate functional restrictions and partial need of outside help, average frequency of epileptic seizures despite anticonvulsant therapy, moderate aphasia; and grade " 5 ", lasting neurological disturbances resulting in severe functional restriction and complete need of outside help, high frequency of epileptic seizures despite of anticonvulsant therapy, severe aphasia. The study included a total of 1681 operations at 1530 patients. The postoperative neurological disturbances occurred in $7.7 \%(n=119)$ of patients. All postoperative neurological disturbances could be easily classified according to the CPNC. The ranking system was proportional to the length of hospital stay. The CPNC is useful and easily applicable to assess the rates of postoperative neurological disturbances. It can be a comparable instrument in the quality management of neurosurgery.

\section{Introduction}

The ranking systems give an important possibility of comparison and are essential for science and quality management in medicine. A reliable assessment of surgical complications and outcomes is increasingly requested by all involved sides (patients, government, insurance companies, clinicians, and scientists), to improve the quality and calculate the costs of healthcare [8]. For this purpose, it is essential to use a standardized, easily applicable, and comparable classification. An approved and validated classification of neurosurgical complications has been advocated as a "main vehicle" for comparison of surgical results within a center, between different centers, various therapies, and over time [14]. The general surgical field has already proposed a "Classification of Surgical Complications" by Clavien \& Dindo. This standardized classification is nowadays widely applied in various surgical disciplines, and it is declared as an effective instrument for quality management $[6,10,12,13,17,19,21,23,26]$. The lack of such standardized classification is a subject of discussion over a long time in the neurosurgical field. The experiences with the Clavien-Dindo classification of surgical complications in neurosurgery have shown an insufficiency for describing the severity of postoperatively occurred neurological disturbances, which are frequent in neurosurgery.

The neurosurgical literature gives until now only simple and less precise classifications as "intra-/postoperative" and "minor/major" complications [15]. It was already proposed by Landriel Ibañez an "A new classification of complications in neurosurgery" based on treatment need to treat the 
complications (Fig. 1) [14]. This classification is very similar to the Clavien-Dindo classification of surgical complications and gives no possibility to rank the postoperatively occurred neurological disturbances. Furthermore, a classification based only on treatment has been designated as not adequate [20].

A classification of neurosurgical complications approved and validated by the entire scientific community has not been developed until now [2].

We aimed to design a classification that could include all possible postoperative occurred neurological disturbances and prove its validity to be comparable and easily used in every main and sub-specialties of neurosurgery.

In this paper, we propose a new classification of postoperative neurological complications (CPNC).

\section{Methods And Definitions Of Complications}

\section{Definition of Complication}

There are overall three basic definitions of negative outcomes in the literature: complication, failure to cure, and sequelae $[5,8]$. Our field of interest included, in this case, only "complication" as a reason for adverse outcomes. In several scientific groups/wards are still debating concerning a universal definition of "complication" [1, 2]. Clavien and Dindo defined a complication as any deviation from a normal postoperative course [8]. Sokol and Wilson suggested the following definition: "A surgical complication is any undesirable, unintended, and direct result of an operation affecting the patient, which would not have occurred had the operation gone as well as could reasonably be hoped" [22].

We defined a "postoperative neurological complication" as any postoperatively occurred neurological disturbances directly or indirectly related to undertaken surgery.

\section{Classification of Postoperative Neurological Complications (CPNC)}

After many discussions and various designs of classification, we chose to concentrate on the type/character and severity of functional restriction due to postoperatively occurred neurological complications. So we designed a Classification with a total of 7 grades commencing from grade " 0 " for no neurological disturbances to grade "6" for coma/vegetative state/demise. The classification is presented in Fig. 2.

Grade 1 neurological complications were defined as during the hospital stay, completely recurrent postoperative occurred neurological disturbances with or without any additional therapy. This grade also includes a single epileptic seizure without the need for further anticonvulsant therapy. Grade 2 includes lasting neurological disturbances without resulting in any functional restriction and seizure freedom under antiepileptic therapy. Grade 3 complications were defined as lasting neurological disturbances resulting in slight functional restrictions. The affected patients require the assistance of some tools but 
do not need outside help. This grade also includes rare epileptic seizures despite anticonvulsant therapy and slight aphasia. Grade 4 complications were defined as lasting neurological disturbances resulting in moderate functional restrictions. The affected patients partially depend on outside help. Epilepsy with an average frequency of seizures despite anticonvulsant therapy and moderate aphasia (e.g., significant restriction of communication) are also included.

Grade 5 neurologic complications were defined as lasting neurological disturbances resulting in severe functional restriction, including a high frequency of epileptic seizures despite anticonvulsant therapy and severe aphasia (e.g., global aphasia). The affected patients depend entirely on outside help.

The severity of epilepsy and aphasia were ranked according to the degree of disability according to the "Pension Medical Ordinance" of the "German Federal Ministry of Labour and Social Affairs" [3].

As it is known, a recovery of neurological disturbances and consequently of functional restriction can take a long time [11]. The neurological status on the day of discharge from acute care hospital mostly is not a final result and can be significantly improved due to medical rehabilitation [25]. Therefore we decided to establish a suffix " $r$ " for defining the long-term outcome. It should give a possibility to compare the short and long-term outcomes, as well as the end results of surgery between different therapies and centers.

\section{Validation of the CPNC in a Cohort of 1530 Patients}

We retrospectively analyzed a period of care between June 2017 and December 2019 in our neurosurgical department. The discharge reports and patient charts of all 1530 patients were studied. The patients with neurological complications were observed in the follow-up period. The evaluated neurological complications were classified according to the CPNC from two independent raters (neurosurgeons of our department). The possible correlations between the various grades of complications and the length of hospital stay (LOS) in this large cohort of patients were also assessed. The length of stay was calculated from the day of initial surgery to the discharge, with the addition of further hospitalizations due to late postoperative complications.

\section{Statistical Analyses}

In the cohort of patients, the correlations between the complication grades and the length of stay were statistically analyzed using two-sided Spearman's rank correlation coefficients. The agreement between the raters was statistically analyzed using Cohen's kappa coefficient.

A $p$-value $<0.05$ was assessed as statistically significant. The statistics were done using the $\mathrm{R}$ for Mac OS X Version 3.3.2 and Microsoft Excel 2011 program. Data were expressed as mean \pm SD, rates, or numbers.

\section{Results}


The study included a total of 1681 operations at 1530 patients. It included $1158(68,8 \%)$ operations on the spine, $46(2,7 \%)$ on the peripheral nervous system, and $477(28,4 \%)$ cases of cerebrovascular surgery. The postoperative neurological disturbances occurred in $7.7 \%(n=119)$ of patients. Therefore both raters sorted a total of $92,3 \%(n=1411)$ of cases as grade 0 . Grade 1 complications were recorded from both raters in 2,68\% $(n=41)$ of cases. Grade 2 complications were recorded in 2,68\% $(n=41)$ of cases from one rater and in 2,55\% $(n=39)$ of cases from another rater. Grade 3 complications were assessed from both raters in $0,98 \%(n=15)$ of patients. Grade 4 complications were recorded from one rater in $0,58 \%(n=9)$ and from another rater in $0,92 \%(n=14)$ of cases. Grade 5 complications were assessed from one rater in $0,39(n=6)$ and from one rater in $0,32 \%(n=5)$ of patients. Grade 6 complications were recorded from both raters in $0,46 \%(n=7)$ of cases (Fig. 3). The agreement between the raters was statistically "almost perfect," according to Landis and Koch, with $\boldsymbol{k}=0,94$ (Cohen's kappa coefficient).

The classification of complications significantly correlated with the lengths of the hospital stay $(r s=0.85$, $p$ (2-tailed $)=0.0137$ Spearman rank correlation test). A median length of Hospitalisation in patients without complication was 7,5 days (SD: 4,9042). The patients with grade 1 complications had a mean length of stay of 10,7 days (SD: 7,47), those with grade 2 complications had LOS of 11,3 days (SD: 9,05), those with grade 3 complications - 20 days (SD: 12,27), those with grade 4 complications had LOS of 18,3 days (SD: 7,69), those with grade 5 complications had a LOS of 17 days (SD: 5,02) and those with grade 6 complications had a LOS of 22 days (SD: 14,61) (Fig. 4).

A total of $80,6 \%(n=72)$ of patients with postoperative complications $(n=119)$ could be observed in the follow-up period. The mean time of postoperative observation in this study was 168,80 days (SD: $142,61)$. A total of $7(5,8 \%)$ patients died due to postoperative complications. There were a total of 30 patients with initial CPNC grade 1 postoperatively observed. 28 patients of them were rated as grade 0 r, and 2 of them as grade 2r. From a total of observed 31 patients with CPNC grade 2, 19 patients showed no changes and were rated as CPNC grade $2 r, 11$ patients had a total reconvalenscence of neurological disturbances and were rated as grade $1 r$, only 1 patient had a worsening of functional restriction and was rated as grade 3 r. From observed 9 patients with grade 3 complications showed 3 patients a stable, functional restriction, 2 patients had a slight improvement and were ranked as grade $2 r$, and 1 patient had a worsening of the functional restriction in the follow-up period and was ranked as grade $4 \mathrm{r}$. Both observed patients with CPNC grade 4 had a slight improvement of functional restriction and were rated as grade 3 r. An observed patient with CPNC grade 5 showed also an improvement and was graded as 3 r. The changes of the postoperatively occured functional restriction are shown in the figure 5 .

\section{Discussion}

The modern, evidence-based medicine requests an increasing demand for quality management. The improvement of quality, in great part, depends on the assessment of negative outcomes. Surgical complications are a major factor in increasing the costs of healthcare $[9,16]$. The need for a classification of complications approved by the scientific community, allowing a comparison of different series of patients, is widely advocated in the neurosurgical society $[2,14]$. 
Despite many trials, the definition of "surgical complication" remains controversial in some scientific groups $[2,5,8,14,22,24]$. The current contention point in the neurosurgical society seems to be the "sequelae" $[2,4]$. According to the oxford dictionary, "sequelae" is defined as "A condition which is the consequence of a previous disease or injury." [18]. In general surgery, a "sequelae" is defined as an "aftereffect" of surgery, for example, an "inability to walk after an amputation of the leg" [8]. In consideration of the above-mentioned, Clark and Spetzler suggested that any, because of surgery complexity expected, postoperatively occurred neurological disturbances should not be assessed as a complication [4]. "For example, a hemiparesis occurring to a patient operated for a large arteriovenous malformation (AVM) located in the motor cortex will be classified as a sequela, whereas the same neurological deficit occurring after the removal of a frontal AVM reaching the Silvian fissure (secondary, that is, to coagulation of a middle cerebral artery branch feeding the corticospinal bundle) should be considered as a complication" [2].

This example of AVM surgery does not seem to be fully comparable to the amputation of the leg. Because of the complexity of the brain's functional structure, there is almost impossible to surely predict the exact extend of postoperative neurological deficits, like the result of leg amputation, excluding the socalled "awake brain surgery" or lobotomy after fiber tracking. However, the evaluation of postoperative occurred expected disturbances seems to be clearly more oriented to self-development and improvement of quality than a camouflage of postoperative occurred neurological deficits under the term of "sequelae."

Obviously, fairness can not involve a direct comparison between the outcomes of AVM and Meningioma surgery, or procedures in eloquent and non-eloquent areas. For this purpose, an additional detailed ranking system of the complexity of neurosurgical procedures could help.

The earlier classifications of surgical complications, which have been applied in neurosurgery, were oriented to the general surgical complications and the treatments necessary to treat them, disregarding the neurological disturbances similar to general surgery $[2,14,15]$. The main difference between neurosurgery and other surgical disciplines is surely the frequency and variety of postoperative neurological disturbances. In principle, the severity of postoperative occurred neurological symptoms frequently defines the outcome of neurosurgical treatment and the patient's quality of life.

Creating the CPNC were discussed several designs of ranking system assessing the postoperative neurological complications:

1. A precise ranking system describing every functional or mental ability of patients and summarizing them in numerical parameters pre-and postoperatively gave a concrete score of worsening of concrete neurological deficit. Still, it did not show an influence of the complication on the affected patient's limitations in daily life. Such classification also seemed to be too multifactorial and complex for use in daily clinical practice.

2. A classification concentrating only on daily life activities of patients, similar to the "Karnofsky index" or "SF-36," seemed to be too imprecise describing the neurological disturbances and gave no 
possibility for ranking the slight neurological deficits. For example, a postoperatively occurred, leftsided L5 - hypesthesia or peroneal palsy by a patient with high-grade hemiparesis on the same side preoperatively, would not cause any additive restriction of daily life activities. Consequently, these complications would be ignored by such a ranking system.

3. A ranking system based on the treatments necessary to treat the complications appeared to be neglected for the end results of neurosurgical procedures. The inadequacy of such classifications to rank the neurosurgical complications has been already reported in the literature $[2,20]$.

Regarding the above mentioned, on the one hand, if we consider the great variety of possible postoperative disturbances, there is an obvious need to generalize or uniting them corresponding to functional restriction to make the classification reproducible and practicable in daily clinical practice. On the other hand, the type, severity, and persistence of neurological complications seems to be also very important to assess the outcome of neurosurgical procedures.

Consequently, the CPNC, as a classification based on type, persistence, and severity of postoperative occurred neurological complications and, at the same time, generalizing them into functional restrictions, seems to have an opportunity of success.

An attempt to validate the new proposal of classification by correlating the grades of complications with the severity of postoperative neurological disturbances was made in a cohort of 1530 patients. The assessed 1680 operations included all sections of the nervous system. Every recorded neurological complication could be easily classified according to the CPNC. The correlation between the CPNC grades and the length of hospital stay, as "a useful parameter of the severity of a complication" $[6,8,9]$, was evident in this article. The simpleness and reproducibility of the proposed classification was statistically validated by the "almost perfect" agreement between the raters with $\boldsymbol{k}=0,94$ (Cohen's kappa coefficient).

Obviously, the fact that improvement of neurological disturbances can take a long time $[11,25]$ and the neurological state at the time of discharge from acute care hospital mostly is not a final result, leads to the requirement of evaluation of neurological state after completed rehabilitation. For this purpose, the suggested suffix "r" appears to be useful for observation of neurological complications in the follow-up period and determination of the final outcome.

With a goal of success for the proposed classification, it is necessary to be approved by the scientific community. For this purpose should be undertaken further multicentral and preferable international studies.

We can recommend the CPNC as an easily applicable and comparable instrument in the quality management of every main and sub-specialties of neurosurgery. The broad implementation of this classification into neurosurgical literature may facilitate the evaluation and comparison of outcomes among different surgeons, centers, and therapies. 
With a goal of success for the proposed classification, it is necessary to be approved by the scientific community. For this purpose should be undertaken further multicentral and preferable international studies.

\section{Declarations}

Funding: This work did not receive any grant from funding agencies in the public, commercial, or not-forprofit sectors.

Conflicts of interest/Competing interests: We certify that the authors have no affiliations with or involvement in any organization or entity with any financial interest (such as honoraria; educational grants; participation in speakers' bureaus; membership, employment, consultancies, stock ownership, or other equity interest; and expert testimony or patent-licensing arrangements), or non-financial interest (such as personal or professional relationships, affiliations, knowledge or beliefs) in the subject matter or materials discussed in this manuscript.

Availability of data and material: The materials included in the manuscript, including all relevant raw data, may be made freely available to any researchers who wish to use them for non-commercial purposes, while preserving any necessary confidentiality and anonymity

Code availability Not applicable

Authors contributions: All authors attest that they meet the current International Committee of Medical Journal Editors (ICMJE) criteria for Authorship.

Ethics approval: Approved by the ethics committee of the General Medical Council of Westphalia-Lippe and the University of Münster (2020-182-f-S)

Consent to participate: Not applicable

Consent for publication Not applicable

\section{References}

1. Bolliger M., Kroehnert J.-A., Molineus F., Kandioler D., Schindl M., \& Riss P. (2018). Experiences with the standardized classification of surgical complications (Clavien-Dindo) in general surgery patients. European Surgery, 50(6), 256-261.

2. Brock S., Saleh C., Zekaj E., \& Servello D. (2016). How to compare clinical results of different neurosurgical centers? Is a classification of complications in neurosurgery necessary for this purpose. Surgical neurology international, 7(Suppl 20), S565.

3. Bundesministerium für Arbeit und Soziales. (2015). "Versorgungsmedizin-Verordnung. Versorgungsmedizinische Grundsätze ". Stand: September 2015, S. 37-38 
4. Clark JC, Spetzler RF. (2011) Creating a brave world for neurosurgery. World Neurosurgery;75:608-9.

5. Clavien PA, Sanabria JR, Strasberg SM. (1992). Proposed classification of complications of surgery with examples of utility in cholecystectomy. Surgery;111(5):518-26.;

6. Collins T, Daley J, Henderson W, et al. (1999). Risk factors for prolonged length of stay after major elective surgery. Ann Surg:230:251-259.

7. de la Rosette J. J. M. C. H., Opondo D., Daels F. P. J., Giusti G., Serrano Á., Kandasami S. V. et al. (2012). Categorisation of Complications and Validation of the Clavien Score for Percutaneous Nephrolithotomy. European Urology, 62(2), 246-255.

8. Dindo D., Demartines N., \& Clavien P.-A. (2004). Classification of Surgical Complications. Annals of Surgery, 240(2), 205-213

9. Ferraris V, Ferraris S, Singh A. (1998). Operative outcome and hospital costs. J Thorac Cardiovasc Surg. ;115:593-603.

10. Fink C. A., Friedrich M., Frey P. E., Rädeker L., Leuck A., Bruckner T. et al. (2018). Prospective multicentre cohort study of patient-reported outcomes and complications following major abdominal neoplastic surgery (PATRONUS) - study protocol for a CHIR-Net student-initiated German medical audit study (CHIR-Net SIGMA study). BMC Surg ., 18(1), 90.

11. Haselbach D, Renggli A, Carda S, Croquelois A. (2014). Determinants of neurological functional recovery potential after stroke in young adults. Cerebrovasc Dis Extra;4(1):77-83. Published 2014 Apr 17.

12. Howard R., Yin Y. S., McCandless, L., Wang, S., Englesbe, M., \& Machado-Aranda, D. (2018). Taking Control of Your Surgery: Impact of a Prehabilitation Program on Major Abdominal Surgery. J Am Coll Surg.

13. Kushiyama S., Sakurai K., Kubo N., Tamamori Y., Nishii T., Tachimori A. et al. (2018). The Preoperative Geriatric Nutritional Risk Index Predicts Postoperative Complications in Elderly Patients with Gastric Cancer Undergoing Gastrectomy. In Vivo, 32(6), 1667-1672.

14. Landriel Ibanez FA, Hem S, Ajler P, Vecchi E, Ciraolo C, Baccanelli M, et al. (2011). A new classification of complications in neurosurgery. World Neurosurg;75:709-15.

15. Lebude B, Yadla S, Albert T, Anderson DG, Harrop JS, Hilibrand A, et al. (2010). Defining "complications" in spine surgery: Neurosurgery and orthopedic spine surgeons' survey. J Spinal Disord Tech ;23:493-500.

16. Mason L, Garcia A. (1984). Hospital costs of surgical complications. Arch Surg. ;119:1065-1066.

17. Mothes A. R., Schlachetzki A., Nicolaus K., Vorwergk J., Lehmann T., Radosa M. P. et al. (2018). LAVH superior to TVH when concomitant salpingo-oophorectomy is intended in prolapse hysterectomy: a comparative cohort study. Arch Gynecol Obstet.

18. Oxford English Dictionary. Sequela. Retrieved 2017-12-31

19. Perrouin-Verbe M. A., Léon P., Denys P., Mongiat-Artus P., Chartier-Kastler E., \& Phé V. (2018). Longterm functional outcomes of augmentation cystoplasty in adult spina bifida patients: A single-center 
experience in a multidisciplinary team. Neurourol Urodyn.

20. Schiavolin S., Broggi M., Acerbi F., Brock S., Schiariti M., Cusin A. et al. (2015). The Impact of Neurosurgical Complications on Patients' Health Status: A Comparison Between Different Grades of Complications. World neurosurgery, 84(1), 36-40.

21. Shang Y., Guo C., \& Zhang D. (2018). Modified enhanced recovery after surgery protocols are beneficial for postoperative recovery for patients undergoing emergency surgery for obstructive colorectal cancer: A propensity score matching analysis. Medicine (Baltimore) , 97(39), e12348.

22. Sokol DK, Wilson J. (2008). What is a surgical complication? World J Surg ;32:942-4.

23. Van der Sluis P. C., van der Horst S., May A. M., Schippers C., Brosens L. A. A., Joore H. C. A. et al. (2018). Robot-assisted Minimally Invasive Thoracolaparoscopic Esophagectomy Versus Open Transthoracic Esophagectomy for Resectable Esophageal Cancer: A Randomized Controlled Trial. Ann Surg.

24. Veen MR,etal. (1999) Recordingandclassificationofcomplica- tionsinasurgicalpractice. Eur J Surg. ;165(5):421-4. discussion 425.

25. Verhofste BP, Glotzbecker MP, Hresko MT, Miller PE, Birch CM, Troy MJ, Karlin LI, Emans JB, Proctor MR, Hedequist DJ. (2019). Perioperative acute neurological deficits in instrumented pediatric cervical spine fusions. J Neurosurg Pediatr. Aug 16:1-11.

26. Zattoni D., Montroni I., Saur N. M., Garutti A., Bacchi Reggiani M. L., Galetti C. et al. (2018). A Simple Screening Tool to Predict Outcomes in Older Adults Undergoing Emergency General Surgery. J Am Geriatr Soc.

\section{Figures}

\begin{tabular}{|l|l|}
\hline grade I & $\begin{array}{l}\text { any non-life-threatening complications treated without invasive } \\
\text { procedures }\end{array}$ \\
\hline grade II & $\begin{array}{l}\text { complications requiring invasive management such as surgical, } \\
\text { endoscopic, and endovascular procedures }\end{array}$ \\
\hline grade III & $\begin{array}{l}\text { life-threatening adverse events requiring treatment in an intensive care } \\
\text { unit (ICU) }\end{array}$ \\
\hline grade IV & deaths as a result of complications \\
\hline
\end{tabular}

Figure 1

A new classification of complications in neurosurgery (Landriel Ibañez FA) 


\begin{tabular}{|l|l|}
\hline \multicolumn{2}{|c|}{ Classification of Postoperative Neurological Complications } \\
(CPNS)
\end{tabular}

Figure 2

CPNC-Classification of postoperative neurological Complications 


\section{Complications according to the CPNC $(n=119)$}

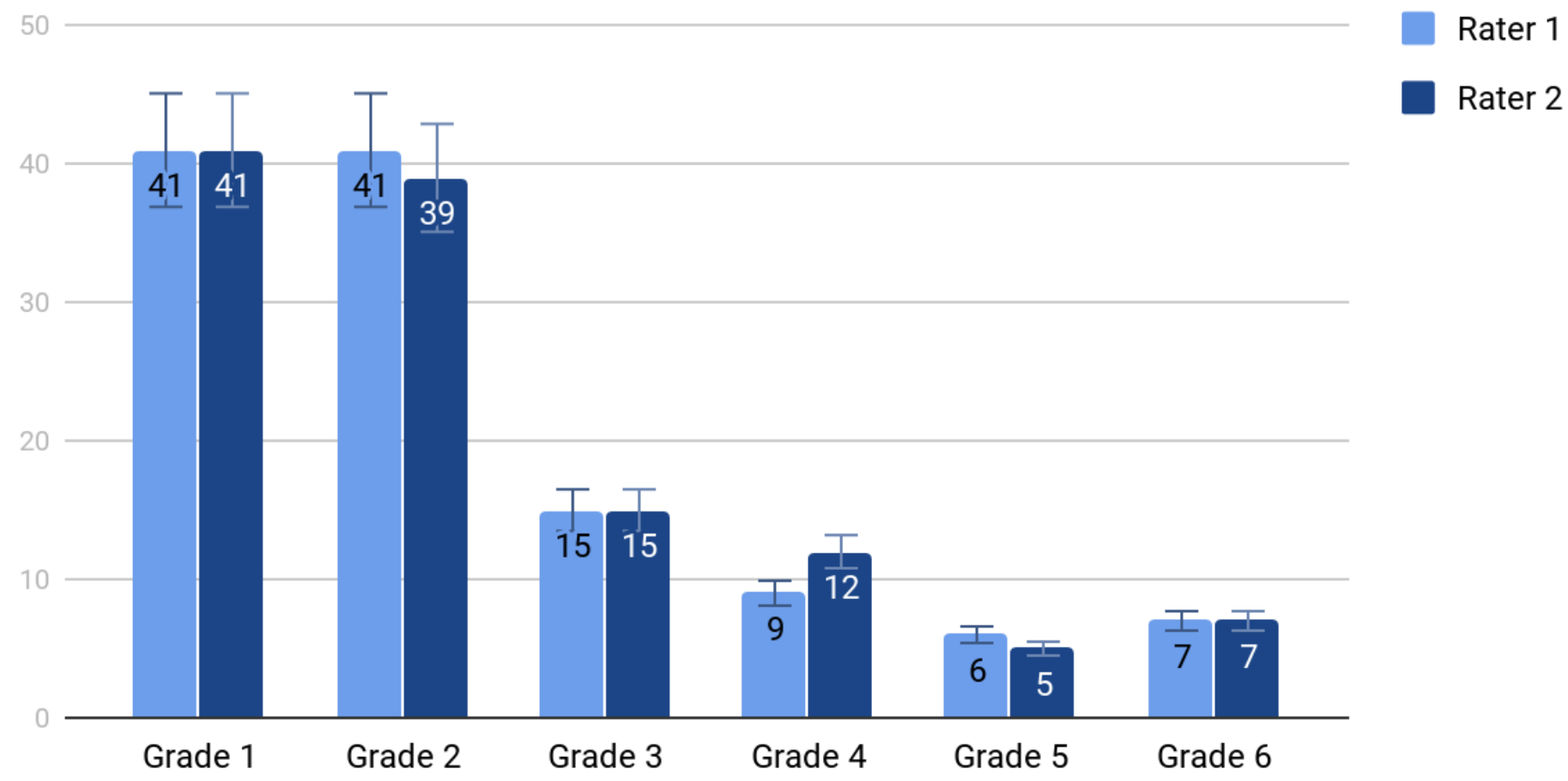

Figure 3

Complications according to the CPNC 


\section{Correlation of LOS with CPNC}
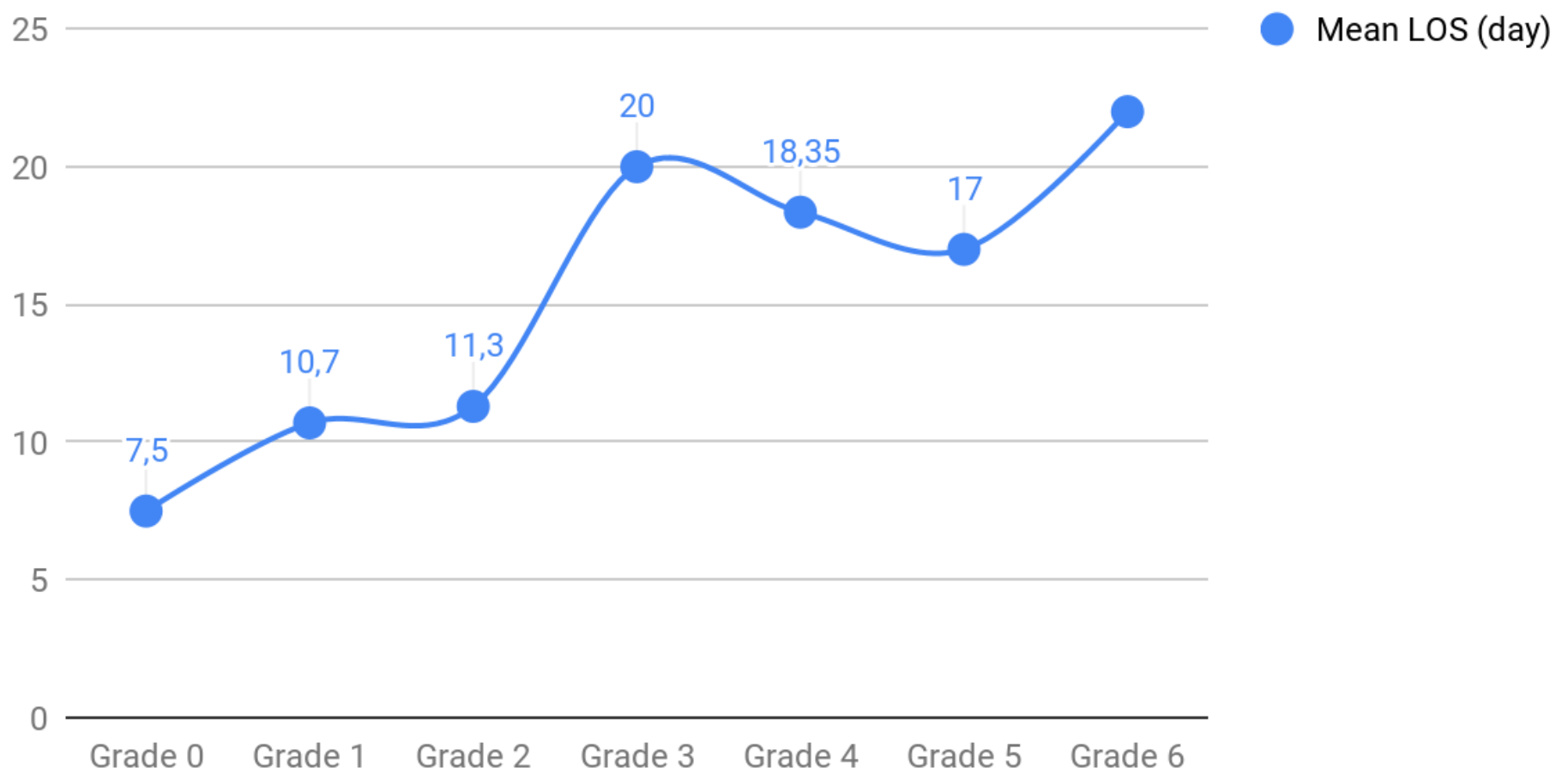

Figure 4

Correlation of LOS with CPNC 


\section{Changes of the functional restriction in the follow up period}

- $\mathrm{CPNCr}$

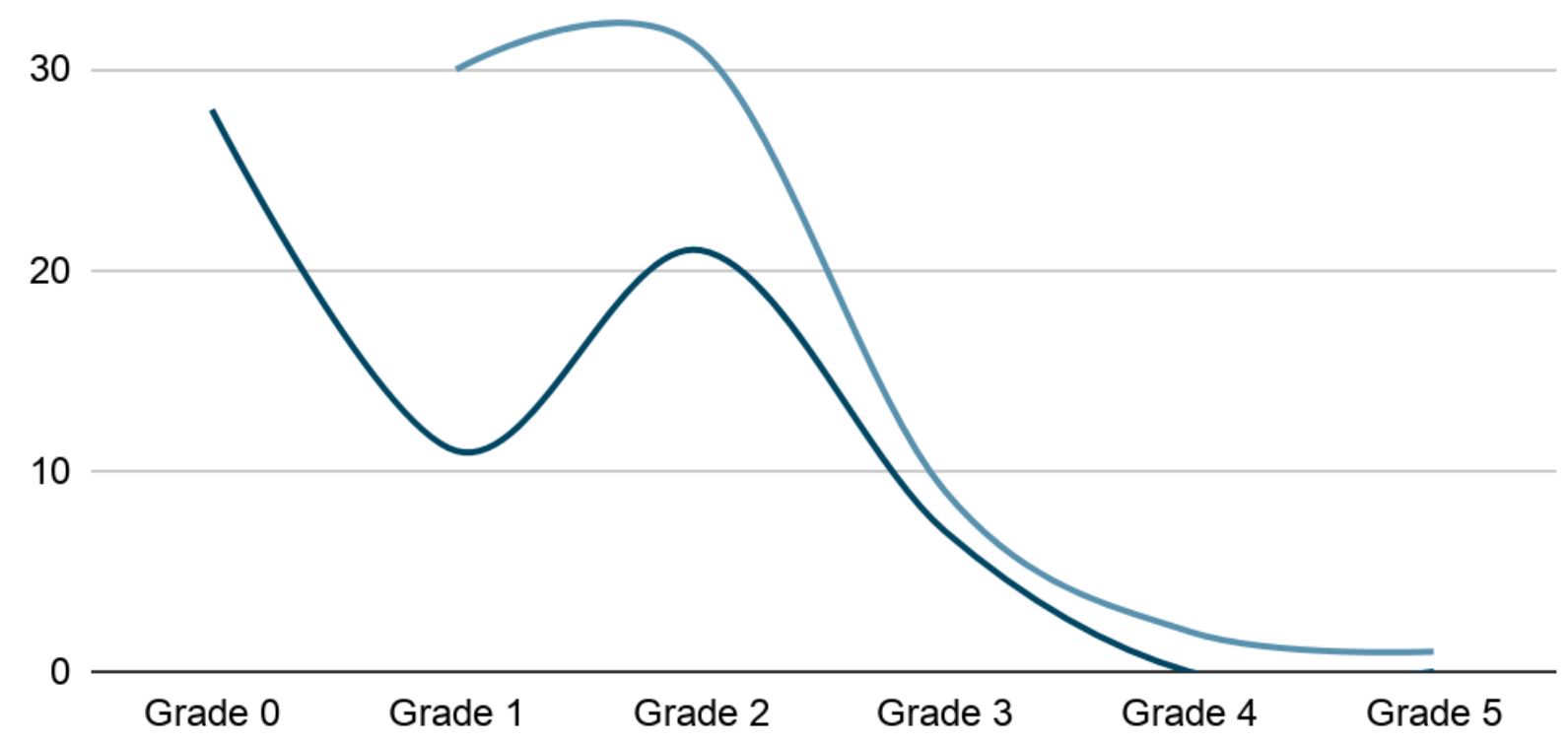

Figure 5

Changes of the functional restriction in the follow-up period 